\title{
¿PODEMOS HABLAR DE ALIMENTOS \\ FUNCIONALES EN CHILE?
}

\author{
CAN WE TALK ABOUT \\ FUNCTIONAL FOODS IN CHILE?
}

\author{
Mariane Lutz R. \\ Centro de Investigación y Desarrollo de Alimentos Funcionales CIDAF, \\ Universidad de Valparaíso. Valparaíso, Chile.
}

\begin{abstract}
Although Chile lacks a formal regulatory definition for functional foods, the term is currently used to designate foods that exert beneficial properties for health due to their content of bioactive ingredients. The Chilean food legislation includes a list of 18 health claims that may be associated to the consumption of a number of foods that exert beneficial effects, all based on sound scientific support. The international food legislation is increasingly demanding the food industry to validate health claims through a series of suitable preclinical (in vitro, in vivo) and clinical studies in order to substantiate the claimed bioactivity of functional food ingredients. An example of the complexity of this requirement is the case of polyphenols, bioactive for which a myriad of beneficial effects have been demonstrated in vitro, although their clinical validation still represents a challenge. Adefinition of functional foods should be established in the Chilean food regulation in order to protect consumers.
\end{abstract}

Key words: Functional food, bioactive compounds, healthy claims, food legislation, validation.

Este trabajo fue recibido el 2 de Agosto de 2011 y aceptado para ser publicado el 1 de Abril de 2012.

\section{NORMATIVA EN RELACIÓN A ALIMENTOS FUNCIONALES Y MENSAJES SALUDABLES}

Chile no cuenta con una definición formal del término "Alimentos Funcionales" (AF), establecida en la normativa alimentaria vigente. Sin embargo, esta nomenclatura es utilizada con gran frecuencia y en forma creciente en los sectores de la industria agroalimentaria, la academia, los profesionales relacionados con alimentos, nutrición y salud. Ha pasado a formar parte del lenguaje de los consumidores e incluso se cuenta con concursos de fondos públicos que financian investigaciones científico-tecnológicas específicamente en el tema (como el Programa FONDEF, iniciado en 2010) y se invierten grandes recursos en investigación de mercado asociado a este rubro alimentario; entonces; ¿es válido hablar de AF en Chile?

La legislación chilena no ha incorporado la definición de AF en el Reglamento Sanitario de Alimentos (RSA), pese a que existe un acuerdo extendido entre la mayoría de los países y grupos de países en cuanto a las características que estos productos deben presentar. De hecho, ya en 2005 un Grupo de Expertos que asesoró durante años al Ministerio de Salud chileno en estas materias propuso la siguiente definición de AF para ser incorporada en el RSA: "AF es aquel que tiene uno o más componentes que satisfactoriamente demuestran que afectan beneficiosamente una o más funciones determinadas del organismo, además de sus efectos nutricionales fundamentales, de manera que sean relevantes tanto para mejorar el estado de salud y bienestar y/o la reducción del riesgo de alguna enfermedad. Un AF debe ser un alimento y debe demostrar sus efectos en cantidades que normalmente se consumen en la dieta." Esta definición está basada y es concordante con lo que ha establecido en esta materia ILSI (International Life Sciences Institute) (www.ilsi.org), organismo que incluye a empresas, academia, legisladores y consumidores y que ha mantenido una actividad permanente de asesoría en materia de AF en los países de Europa, América, Asia y Oceanía (1).

A nivel nacional, ILSI Surandino ha mantenido un interés permanente por el tema y organizado una serie 
periódica de seminarios orientados en los alimentos e ingredientes funcionales en la última década.

Esta situación es bastante similar en otros países; así la legislación europea no ha definido hasta el momento el término AF, no obstante la Agencia Europea de Inocuidad Alimentaria (www.efsa.europa.eu) considera que hace referencia a alimentos que se consumen como parte de una dieta normal y contienen componentes biológicamente activos, que ofrecen beneficios para la salud y reducen el riesgo de sufrir enfermedades (2). Un requisito fundamental es que los $\mathrm{AF}$ sean propiamente alimentos, es decir, que se consuman como parte de la dieta habitual y no se presenten en formas farmacéuticas que contienen altas dosis de determinados compuestos, generalmente en la forma de concentrados que se presentan como cápsulas, aislados o extractos, los que en estricto sentido corresponden a productos "nutracéuticos".

Una salida a esta falta de normativa explícita está dada por la existencia de los denominados "Mensajes Saludables" o declaraciones de propiedades saludables (Health Claims) Segín el Codex Alimentarius (www. codexalimentarius.net), programa conjunto de la Organización de las Naciones Unidas para la Agricultura y La Alimentación (FAO) y la Organización Mundial de la Salud (OMS), que se encarga de establecer normas alimentarias, y que constituye el principal referente en normativa alimentaria a nivel mundial, los Mensajes Saludables corresponden a "cualquier representación que declara, sugiere o implica que existe una relación entre un alimento, o un constituyente de dicho alimento, y la salud" (3).

La empresa alimentaria puede hacer declaraciones de propiedades saludables en relación a sus productos, las que el consumidor podrá conocer para realizar una compra informada. Los 18 mensajes saludables actualmente vigentes en Chile fueron aprobados por el Ministerio de Salud mediante la Resolución Exenta $\mathrm{N}^{\circ} 764 / 09$ (listado disponible en www.minsal.cl). Los mensajes saludables, dirigidos al consumidor, detallan las propiedades de nutrientes y factores alimentarios cuyo consumo se relaciona con la salud, estableciendo los requisitos que debe cumplir un producto alimentario para poder hacer uso de ellos (4).

La inclusión de un mensaje saludable en el listado nacional se fundamenta en los antecedentes que los industriales interesados hacen llegar, conjuntamente con la solicitud de incorporar el mensaje, al Ministerio de Salud, División de Políticas Públicas. Con posterioridad, estos antecedentes, que corresponden fundamentalmente a los principales estudios que validan las propiedades beneficiosas para la salud que se atribuye al nutriente o factor alimentario, son analizados por expertos en estas materias, aprobando o no la solicitud y estableciendo las condiciones de uso del mensaje y su marco o contexto. Este procedimiento es bastante similar al que se aplica en numerosos países, como es el caso de la Food and Drug Administration (FDA) de los Estados Unidos, entidad que ha publicado guías con instrucciones para que las empresas elaboren las solicitudes de mensajes y explican los fundamentos científicos a considerar por los paneles de expertos $(5,6)$.

La carencia de una normativa nacional clara en relación a la definición de los AF, que permita aplicar el término a determinados productos que cumplan con ciertos requisitos, ha llevado a situaciones confusas. Por ejemplo, en algunos países se denomina como AF a alimentos comunes que contienen niveles destacados de ciertos nutrientes como minerales, vitaminas o ácidos grasos, así como a los alimentos a los que se han añadido sustancias biológicamente activas, como diversos fitoquímicos u otros agentes como los probióticos, cultivos de microorganismos vivos. Entonces, ¿todos los alimentos, por su contenido de nutrientes, son funcionales? Los productos tradicionales, como las frutas, las verduras, los cereales integrales, las leguminosas, que contienen compuestos químicos bioactivos que pueden resultar beneficiosos para la salud ¿son todos funcionales?; y el agua, indispensable para la salud ¿es funcional? Las preguntas suman y siguen. Esta falta de claridad probablemente se relaciona en gran medida con el término "funcional", que alude a que algunos componentes del alimento ejercen una acción en el organismo, modulando funciones específicas: si así fuera, naturalmente todos los alimentos serían funcionales por el simple hecho de contener ya sea agua, vitaminas, minerales, grasas, proteínas, hidratos de carbono. Sin duda, no es un término afortunado y puede inducir con gran facilidad al consumidor a una gran confusión. Cuando Japón introdujo, en la década de 1980, el concepto de "alimentos para necesidades específicas de salud” (FOSHU), quedó establecido que el objetivo fundamental de estos productos era reducir el riesgo de enfermedades crónicas. Sin embargo, este objetivo se ha ampliado bastante, ya que en el mercado internacional se encuentran disponibles los más diversos productos alimentarios cuya promoción abarca propiedades nutritivas, saludables, preventivas e incluso curativas (pese a que este último es un aspecto que ninguna legislación alimentaria permite).

Un aspecto clave de los AF es que son, precisamente, ALIMENTOS, por lo que deben ser consumidos en las cantidades propias de una dieta habitual, y no incluyen a los suplementos alimentarios que se ingieren en forma semi-purificada o purificada y en altas dosis. A modo de ejemplo, no tiene ningún sentido afirmar que una 
infusión de té verde es considerada como un AF sobre la base de que algunos compuestos fenólicos del té (en este caso diversas catequinas) poseen una serie de propiedades bioactivas, ya que las cantidades a consumir para que se logre evidenciar los efectos saludables es muy superior a una ingesta "normal" de la infusión, aún con la variabilidad que esto representa. Similar es el caso de la gran mayoría de las frutas, algunas de las cuales incluso han sido denominadas y promocionadas como "superfrutas" por su elevado aporte de fitoquímicos saludables, principalmente en la forma de antioxidantes (como es el caso de la granada, el maqui, el goji, el acai); sin embargo, esta cualidad de ser alimentos saludables es precisamente la que les da su valor en la alimentación habitual, al aportar nutrientes y no nutrientes bioactivos, pero no sería adecuado denominar como AF al tomate porque aporta licopenos, la naranja porque aporta vitamina $\mathrm{C}$ o la palta porque aporta ácido oleico, entre cientos de ejemplos de este tipo.

La diferencia entre estos alimentos, que sin duda alguna son SALUDABLES y cuyo consumo debe ser estimulado en la población de todas las edades, y los $\mathrm{AF}$, es que en estos últimos las propiedades beneficiosas para la salud deben ponerse en evidencia a un nivel de consumo del alimento que corresponde a las condiciones de ingesta habitual.

\section{VALIDACIÓN DE LOS EFECTOS SALUDABLES DE ALIMENTOS E INGREDIENTES FUNCIONALES}

La fundamentación de las propiedades beneficiosas de los AF constituye un tema complejo, que cada vez está siendo más exigido a la empresa alimentaria. $\mathrm{Si}$ el consumidor está dispuesto a pagar más por un valor agregado del producto que escoge, que en el caso del AF se relaciona con un beneficio para su salud al reducir factores de riesgo de enfermedades, el productor del AF debe hacerse responsable de acreditar que este beneficio, declarado a través de un mensaje saludable, está llegando al consumidor $(7,8)$. Para ello, es necesario recurrir a la validación de las propiedades saludables mediante ensayos de distinta naturaleza que abarcan desde el conocimiento de cuáles son los compuestos químicos que ejercen la acción esperada (su identificación química, la prueba de que se encuentran en el producto en las cantidades establecidas), ensayos de laboratorio que abarcan pruebas in vitro en modelos tales como cultivos de células, tejidos u órganos, ensayos in vivo en animales sometidos a dietas de prueba, así como pruebas clínicas realizadas en individuos sanos que consumen el AF de prueba para evidenciar algún efecto mediante el uso de biomarcadores de efecto, pruebas de biodisponibilidad, si el compuesto bioactivo debe distribuirse en el organismo para ejercer su acción, pruebas de su metabolismo (metabolómica), efecto sobre genes (genómica) u otras específicas en cada situación.

Una de las complicaciones de estos ensayos se relaciona con la forma de actuar de muchos compuestos bioactivos, que reducen factores de riesgo de enfermedades de larga data tales como las cardiovasculares, el cáncer o el síndrome metabólico, por lo que es complejo escoger un punto final de un ensayo capaz de detectar el efecto esperado en un tiempo adecuado.

También resulta complejo diseñar estudios relacionados con enfermedades tan multifactoriales como los ensayos de agentes que contribuyan a reducir la obesidad o la hipertensión, para lo cual se debe incluir como individuos de prueba a quienes padecen estas alteraciones metabólicas. No obstante estas complicaciones, las metodologías aplicadas a los ensayos de bioactividad han sido estandarizadas y se ha logrado establecer los requisitos mínimos para que un estudio tenga validez científica. Las agencias reguladoras son las encargadas de analizar, aceptar o rechazar las propuestas de mensajes saludables que se les haga llegar, las que se deben basar en los estándares aceptados de evidencia científica disponibles $(9,10)$.

Un tipo de compuestos bioactivos que representa un gran desafío para la validación de sus propiedades beneficiosas son los compuestos fenólicos. Numerosos estudios epidemiológicos y clínicos han establecido que una ingestión regular de frutas, verduras y jugos naturales se asocia a un menor riesgo de desarrollar enfermedades crónicas no transmisibles $(11,12)$. Los efectos beneficiosos del consumo de estos productos se han asociado tradicionalmente a su elevado contenido de polifenoles, moléculas bioactivas que afectan la dislipidemia y ateroesclerosis, la disfunción endotelial e hipertensión, la activación plaquetaria y trombosis, el proceso inflamatorio asociado a la inducción y desarrollo de enfermedades cardiovasculares, entre otros (13). Ello ha llevado al desarrollo de técnicas de cultivo que inducen que diversas plantas produzcan más compuestos fenólicos de interés y se ha diseñado una gran variedad de alimentos que contienen polifenoles (ifuncionales?) que, presumiblemente, contribuyen a retardar el desarrollo de enfermedades cardiovasculares, cáncer, síndrome metabólico o neurodegeneración, entre otras.

Debido a que el consumo de alimentos aportadores de estos compuestos se asocia con la prevención de enfermedades degenerativas, especialmente asociadas a estrés oxidativo, durante más de una década se ha estudiado los efectos beneficiosos de la ingestión de polifenoles con miras a demostrar la asociación entre su rol 
antioxidante y la prevención del daño oxidativo. Si bien se ha demostrado una alta correlación entre el contenido de compuestos fenólicos y la capacidad antioxidante de frutas, verduras y jugos utilizando diversas formas de medición in vitro de esta propiedad, como los ensayos DPPH, FRAP y ORAC (14), ello no garantiza que esta propiedad sea ejercida in vivo en el organismo.

Para comenzar: ¿De qué compuestos fenólicos se trata? ¿Cuáles son los que se encuentran en determinado alimento y en qué forma se encuentran? Se han descrito más de 8000 compuestos fenólicos diferentes, de diverso tamaño molecular y propiedades físicoquímicas como su solubilidad, y además se pueden encontrar en formas químicas diversas: libres, asociados a azúcares (glicósidos), ligados a componentes de la fibra dietética, entre otras. Cada vegetal posee un patrón de polifenoles que le es característico, el cual a su vez depende de la variedad (genética), condiciones de cultivo, condiciones climáticas, tiempo de cosecha, tratamiento postcosecha.

$\mathrm{Al}$ ser ingeridos los polifenoles, se produce una serie de procesos que dependen de la matriz alimentaria que los contiene y también del medio en el que se encuentran, que es propio de cada individuo y dependiente de su microbiota intestinal (15). En consecuencia, el destino de las moléculas que originalmente se encuentran en el alimento puede variar significativamente entre diferentes sujetos, según la actividad hidrolítica de la flora intestinal, que puede metabolizar los compuestos nativos originando otros que serán más o menos absorbibles a nivel intestinal. En términos generales, la biodisponibilidad de los compuestos fenólicos (aquella parte de lo ingerido que circula en la sangre y está disponible para ejercer sus efectos en los tejidos corporales) es extremadamente baja. Asimismo, los procesos de conjugación que transforman los compuestos ingeridos en otros más polares determinan si su vía de excreción será biliar o urinaria, y sería necesario tener claro si los metabolitos generados poseen o no las propiedades bioactivas esperadas (16-19).

Entonces, ¿es posible validar los AF que contienen polifenoles, sea en forma natural o adicionados como ingredientes funcionales? La respuesta no es simple. Si bien se cuenta con un amplio espectro de ensayos preclínicos que validan diversos efectos saludables de estos compuestos, la situación in vivo es diferente. Su variedad y complejidad estructural es enorme, lo que demanda un conocimiento acabado de los procesos involucrados en su absorción, distribución, metabolismo y excreción, con el fin de establecer cuáles son los biomarcadores de efecto adecuados, así como el conocimiento de los mecanismos de acción involucrados en sus efectos en el orga- nismo (20). A ello han contribuido significativamente los avances en la química analítica de alta sensibilidad, que permite la identificación y cuantificación de múltiples moléculas a niveles cada vez menores.

En los últimos años el desarrollo de las técnicas de la metabolómica ha contribuido a identificar las respuestas de cada sujeto en particular a la ingestión de compuestos bioactivos, logrando una buena aproximación para conocer sus mecanismos de acción $(21,22)$, así como el desarrollo de técnicas de ensayos a nivel molecular, genético y preclínicos que permiten buscar los mecanismos de acción específicos de los compuestos bioactivos. El problema aún no resuelto se relaciona con este aspecto, ya que durante años los efectos beneficiosos de los compuestos fenólicos se atribuyeron directamente a su capacidad antioxidante; sin embargo, hoy en día se acepta que sus formas de actuar son indirectas, y pueden ejercerse incluso a concentraciones tisulares extremadamente bajas, a través de la inducción de señales celulares y/o expresión de genes relacionados con la protección del organismo mediante procesos asociados al desarrollo de patologías como las cardiovasculares, cáncer o neurodegeneración $(23,24)$. Buscando una relación causal entre la capacidad antioxidante de los compuestos fenólicos ingeridos, el daño oxidativo y la reducción de riesgo de enfermedades cardiovasculares, Hollman y cols (25) aplicaron los criterios europeos de validación $(26,27)$ y no lograron sustentar los mensajes saludables asociados a sus efectos antioxidantes. Este ejemplo pone en evidencia que la validación de los efectos saludables de los polifenoles, que permitan establecer declaraciones de propiedades beneficiosas asociadas a su consumo, aún representa un desafío, especialmente debido a que es muy complejo caracterizar el conjunto de compuestos responsables de la bioactividad.

¿Existe algún mensaje saludable aprobado relacionado con polifenoles en alimentos? La Comisión Europea solicitó al Panel de Expertos en Productos Dietéticos, Nutrición y Alergias que entregara una opinión con base científica acerca de un listado de propiedades saludables asociadas al consumo de polifenoles (hidroxitirosol y complejo oleuropeina) en aceitunas (fruto, aguas de descarte de la molienda o aceite de oliva, extracto y hojas de Olea europaea L.), de acuerdo al Artículo 13 del Reglamento europeo (EC) 1924/2006. Las propiedades para las cuales se solicitó establecer mensajes incluyen la protección de la oxidación de las LDL, la mantención de los niveles de colesterol HDL, la mantención de la presión arterial normal, propiedades antiinflamatorias, la contribución a la salud del tracto respiratorio superior, la mantención de la función normal del tracto gastrointestinal y la contribución a las defensas del organismo 
contra agentes externos. Luego de analizar los antecedentes entregados, el Panel de Expertos consideró que los polifenoles en los productos antes señalados, habiendo estandarizado su contenido de hidroxitirosol y derivados (complejo europeina), están suficientemente caracterizados como para sustentar los mensajes solicitados y estableció los requisitos de consumo: para prevenir la oxidación de LDL se recomienda una ingesta de al menos $5 \mathrm{mg}$ diarios de los compuestos bioactivos presentes en el aceite de olivas. La evidencia presentada para los otros mensajes saludables solicitados se consideró insuficiente y no se aprobaron. En consecuencia, el único mensaje aprobado señala que "el consumo de polifenoles del aceite de oliva contribuye a la protección de los lípidos sanguíneos del daño oxidativo" (28).

\section{CONCLUSIÓN}

Sin duda, para hablar con propiedad de los AF en Chile es necesario establecer con claridad los criterios mínimos de aplicación de esta terminología en la reglamentación alimentaria, lo cual favorecerá a todos los interesados: los consumidores, los empresarios y los legisladores, con el apoyo de los investigadores que cuentan con las capacidades para validar las propiedades declaradas en los mensajes saludables. Los consumidores tienen derecho a saber qué compuestos bioactivos están presentes en los AF que escogen consumir, por los cuales están pagando, las cantidades que entrega una porción y la forma en la que debiera ser consumido, como también si estos compuestos de interés son realmente absorbidos y van a ejercer su acción al distribuirse por la circulación sanguínea en el organismo, llegando a los tejidos donde ejercerá su acción o si actúan en forma local en el tracto gastrointestinal, entre otros. El caso de los polifenoles ejemplifica la complejidad y los desafíos que representa esta aproximación a los AF, así como los pasos a seguir cuando se enfrenta el tema de entregar mensajes al consumidor en relación con los atributos saludables de ciertos alimentos en forma responsable y fundamentada, información que le permita elegir con propiedad su alimentación y la de su familia, a la vez que permite acotar las propiedades que describe la publicidad en relación con los alimentos en un marco de responsabilidad ética y social.

\section{RESUMEN}

En Chile no se cuenta con una definición formal del término Alimento Funcional, el que se utiliza para designar alimentos que ejercen propiedades beneficiosas debido a su contenido de ingredientes bioactivos. La reglamentación alimentaria incluye un listado de 18 mensajes o declaraciones de propiedades saludables, los que se asocian al consumo de alimentos que poseen propiedades beneficiosas para la salud, todos ellos basados en evidencia científica. La legislación internacional está demandando cada vez más a la industria la validación de los mensajes saludables a través de estudios preclínicos y clínicos que entreguen una base científica sólida a la bioactividad declarada de los ingredientes funcionales. Un ejemplo de la complejidad del tema es el caso de los polifenoles, para los cuales se ha demostrado una gama de efectos beneficiosos en ensayos in vitro, en tanto que su validación clínica aún representa un desafío. Se plantea la necesidad de definir los criterios de uso del término alimentos funcionales con miras a la protección del consumidor.

Palabras clave: alimentos funcionales, compuestos bioactivos, mensajes saludables, legislación alimentaria, validación.

Dirigir la correspondencia a:

Profesora

Mariane Lutz R.

CIDAF, Facultad de Farmacia,

Universidad de Valparaíso

Gran Bretaña 1093

Valparaíso. Chile.

Fono 032-2508418

Fax 032-2508111

E-Mail: mariane.lutz@uv.cl

\section{BIBLIOGRAFÍA}

1. ILSI. Perspectives on ILSI's International Activities on Functional Foods. Report commissioned by the ILSI Europe Functional Foods Task Force, 2009.

2. EU Parliament. C1 Regulation (EC) No 1924/2006 of the European Parliament and of the Council of 20 December 2006 on Nutrition and Health Claims made on Foods. 2006.

3. Codex Alimentarius. Declaraciones Nutricionales y Saludables (CAC/GL 23-1997). Adoptadas en 1997; Revisadas 2004; Enmendadas 2001, 2008, 2009, 2010. Publicado 2011.

4. Ministerio de Salud. Normas técnicas sobre directrices nutricionales que indica, para la declaración propiedades saludables de los alimentos. Resolución Exenta $N^{\circ} 764 / 09$, Diario Oficial 05.10.09.

5. Food and Drug Administration, US Department of Health and Human Services. 2009. Guidance for Industry: Evidence-Based Review System for the Scientific Evaluation of Health Claims - Final

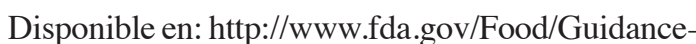
ComplianceRegulatoryInformation/GuidanceDocu- 
ments/FoodLabelingNutrition/ucm073332.htm

6. Food and Drug Administration, US Department of Health and Human Services. 2011. Health Claims Meeting Significant Scientific Agreement (SSA) Disponible en http//www fda gov/Food/LabelngNutrition/LahelClaims/HealthClaimsMeetingSignificantScientificAgreementSSA/default.htm

7. Lutz M. Alimentos funcionales en la prevención de enfermedades crónicas no transmisibles. En: "Aspectos nutricionales y saludables de los productos de panificación”. Lutz M, León AE, eds. Sello Universidad de Valparaíso, 2009, p 39-51.

8. Lutz M, Greco C, Trigo MJ. Aspectos legales en relación con los alimentos funcionales: definiciones y normativa. En: "Aspectos nutricionales y saludables de los productos de panificación". Lutz M, León AE, eds. Sello Universidad de Valparaíso, 2009, p 190-203.

9. American Dietetic Association. Position of the American Dietetic Association: Functional Foods. J Amer Diet Assoc 2009; 109: 735-46.

10. ILSI Europe. Beyond PASSCLAIM - Guidance to Substantiate Health Claims on Foods. Summary Report of a Workshop held in December 2009. Report Series, Belgium, 2010, p. 1-28.

11. Arts I, Hollman P. Polyphenols and disease risk in epidemiologic studies. Am J Clin Nutr 2005; 81: 317S-25S.

12. Erdman J, Balentine D, Arab L, Beecher G, Dwyer JT, Folts J, Harnly J, Hollman P, Keen CL, Mazza G, Messina M, Scalbert A, Vita J, Williamson G, Burrowes J. Flavonoids and heart health. Proceedings of the ILSI North America Flavonoids Workshop. J Nutr 2007; 137: 718S-37S.

13. Fraga CG, Galleano M, Verstraeten SV, Oteiza PI. Basic biochemical mechanisms behind the health benefits of polyphenols. Mol Asp Med 2010; 31: 435-45.

14. Lutz M, Jorquera K, B. Cancino, Henríquez C. Phenolics content and antioxidant capacity of table grape cultivars grown in Chile. J Food Sci 2011; 76: C1088-C1093.

15. Porrini M, Risso P. Factors influencing the bioavailability of antioxidants in foods: A critical appraisal. Nutr Metab Cardiovasc Dis 2008; 18: 647-50.

16. Scalbert A, Williamson G. Dietary intake and bioavailability of polyphenols. J Nutr 2000; 130 :
2073S-85S.

17. Manach C, Scalbert A, Morand C, Rémésy C, Jiménez L. Polyphenols: food sources and bioavailability. Am J Clin Nutr 2004; 79: 727-47.

18. Crozier A, Del Rio D, Clifford MN. Bioavailability of dietary flavonoids and phenolic compounds. Mol Asp Med 2010; 31: 446-67.

19. D’Archivio M, Filesi C, Varì R, Scazzocchio B, Masella R. Bioavailability of the polyphenols: Status and controversies. Int J Mol Sci 2010; 11: 1321-42.

20. Lutz M. Soy Isoflavones as Bioactive Ingredients of Functional Foods. En: Soybean and Health, Hany El-Shemy (Ed.), InTech, 2011, p 329-60.

21. Gibney MJ, Walsh M, Brennan L, Roche HM, German B, van Ommen B. Metabolomics in human nutrition: opportunities and challenges. Am J Clin Nutr 2005; 82: 497-503.

22. Fardet A, Llorach R, Orsoni A, Martin JF, PujosGuillot E, Lapierre C, Scalbert A. Metabolomics provide new insight on the metabolism of dietary phytochemicals in rats. J Nutr 2008; 138: 1282-87.

23. Stevenson DE, Hurst RD. Polyphenolic phytochemicals - just antioxidants or much more? Cell Molec Life Sci 2007; 64: 2900-16.

24. Kampkötter A, Chovolou Y, Kulawik A, Röhrdanz E, Weber N, Proksch P, Wätjen W. Isoflavone daidzein possesses no antioxidant activities in cell-free assays but induces the antioxidant enzyme catalase. Nutr Res 2008; 28: 620-8.

25. Hollman PCH, Cassidy A, Comte B, Heionen M, Richelle M, Richling E, Serafini9 M, Scalbert A, Sies H, Vidry S. The biological relevance of direct antioxidant effects of polyphenols for cardiovascular health in humans is not established. J Nutr 2011; 141: 989S-1009S.

26. Asp NG, Bryngelsson S. Health claims in Europe: new legislation and PASSCLAIM for substantiation. J Nutr 2008; 138: S2010-S1215.

27. Aggett PJ, Antoine JM, Asp NG, Bellisle F, Contor L, Cummings JH, Howlett J, Muller DJ, Persin C, Pijls LT, Reckhemmer S, Tuijtelaars s, Verhagen H. PASSCLAIM: consensus on criteria. Eur J Nutr 2005; 44 (Suppl 1): i5-i30.

28. European Food Safety Authority. EFSA Journal 2011; 9(4): 2033 [25 pp] doن.102903/jefsa 20112033 Disponible en www.efsa.europa.eu/ efsajournal. 